\title{
Metabolism Analysis of Alantolactone and Isoalantolactone in Rats by Oral Administration
}

\author{
Mengyue Wang, Renjie Xu, Ying Peng (D), and Xiaobo Li (iD) \\ School of Pharmacy, Shanghai Jiao Tong University, Shanghai 200240, China \\ Correspondence should be addressed to Xiaobo Li; xbli@sjtu.edu.cn
}

Received 3 August 2017; Accepted 10 December 2017; Published 13 March 2018

Academic Editor: Patricia Valentao

Copyright (C) 2018 Mengyue Wang et al. This is an open access article distributed under the Creative Commons Attribution License, which permits unrestricted use, distribution, and reproduction in any medium, provided the original work is properly cited.

\begin{abstract}
Alantolactone and isoalantolactone are the major active ingredients of Inulae Radix. Their metabolism in vivo and in vitro was investigated by UPLC-Q-TOF-MS for the first time. As a result, nine metabolites in vivo including cysteine conjugates, oxidates, dehydrogenates, and hydrates were detected in rat bile after oral administration. The metabolites produced in vitro by incubation with rat liver microsomes were found to be substantially identical to those detected in vivo. However, no metabolites were detected in the samples of plasma, feces, and urine or in the incubates of gastric juice, intestinal juice, and intestinal bacteria. These results reveal that the liver is the main metabolic organ for alantolactone and isoalantolactone, and the first pass effect of the liver appears to be the reason for the low oral bioavailability of the two lactones.
\end{abstract}

\section{Introduction}

Alantolactone and isoalantolactone, first isolated from Inula helenium [1], are the main active constituents in Radix Inulae, commonly used in a folk medicine called Tu-Mu-Xiang in China [2]. Current literature shows that alantolactone and isoalantolactone possess various antimicrobial [3], antiinflammatory [4], and antitumor activities [5-10] both in vivo and in vitro. A recent study done by the team revealed that alantolactone and isoalantolactone could significantly relieve irritable bowel syndrome (IBS) by regulating neurotransmitters, gastrointestinal hormones, and intestine propulsive movement. This study suggested that alantolactone and isoalantolactone were promising candidates for IBS applications [11].

To further evaluate their potential use as drugs, some key pharmacokinetic parameters were investigated in the previous study. The results demonstrated that the absolute bioavailabilities of alantolactone and isoalantolactone were not high $[12,13]$, indicating that they may undergo metabolism in vivo. However, no metabolism studies on the two lactones have been reported. In the present study, their metabolic profiles in vivo in rats and in vitro were elucidated.

\section{Experimental}

2.1. Chemicals and Materials. Dried roots of Inula helenium L. were purchased from the herbal market in May 2015 in Sichuan Province, China, and identified by Professor Xiaobo Li. A specimen (SJTUIH 20150509) was deposited in the School of Pharmacy, Shanghai Jiao Tong University. HPLCgrade acetonitrile was purchased from Merck Company (Darmstadt, Germany). Petroleum ether $\left(60-90^{\circ} \mathrm{C}\right)$, acetone, dichloromethane, alcohol, and silver nitrate were of analytical grade and purchased from China National Medicines Co. Ltd. (Shanghai, China). Ultra-pure water used in UPLC mobile phase was prepared using a Milli-Q system (Millipore, Bedford, MA, USA).

2.2. Preparation of Alantolactone, Isoalantolactone, and Other Relevant Lactones. Inula helenium roots $(2.1 \mathrm{~kg})$ were extracted by refluxing with $9 \mathrm{~L} 95 \%$ ethanol twice (each $2 \mathrm{~h}$ ). The extraction solution was completely evaporated under reduced pressure to afford a dark brown extractive $(137 \mathrm{~g})$. The extractive was suspended in $1500 \mathrm{~mL}$ water and extracted with dichloromethane $(1000 \mathrm{~mL} \times 3)$. Dichloromethane solution was evaporated under reduced pressure to leave 
behind a brown residue $(58 \mathrm{~g})$. The residue was subjected to silica gel CC $(90 \mathrm{~cm} \times 8 \mathrm{~cm})$ which was eluted with petroleum ether-acetone $(100: 0$ to $95: 5, \mathrm{v} / \mathrm{v})$ to afford 34 fractions (Fr.1-Fr.34). Fr.6 (5.2 g) was further separated by silica gel (containing $10 \%$ silver nitrate) CC $(70 \mathrm{~cm} \times 5.5 \mathrm{~cm})$ eluted with petroleum ether-acetone $(98: 2, \mathrm{v} / \mathrm{v})$ to afford compounds $1(266 \mathrm{mg})$ and $2(183 \mathrm{mg})$. Fr.8 $(847 \mathrm{mg})$ was further purified by silica gel CC $(60 \mathrm{~cm} \times 4.5 \mathrm{~cm})$ eluted with petroleum ether-acetone ( $98: 2$ to $96: 4, \mathrm{v} / \mathrm{v}$ ) to afford compound $3(18 \mathrm{mg})$. Fr.10 $(562 \mathrm{mg})$ was further subjected to silica gel CC $(60 \mathrm{~cm} \times 4.5 \mathrm{~cm})$ eluted with petroleum ether-acetone $(97: 3, \mathrm{v} / \mathrm{v})$ to afford compound $4(53 \mathrm{mg})$. Fr.13 (509 mg) was further purified by silica gel CC $(50 \mathrm{~cm} \times 4.5 \mathrm{~cm})$ eluted with petroleum ether-acetone $(95: 5$ to $92: 8, \mathrm{v} / \mathrm{v})$ to afford compounds $5(22 \mathrm{mg})$ and $6(13 \mathrm{mg})$. Fr.16 $(419 \mathrm{mg})$ was further separated by silica gel (containing $10 \%$ silver nitrate) CC $(50 \mathrm{~cm} \times 4.5 \mathrm{~cm})$ eluted with petroleum ether-acetone $(85: 15, \mathrm{v} / \mathrm{v})$ to afford compounds $7(11 \mathrm{mg})$ and $8(5 \mathrm{mg})$.

\subsection{Animal Experiment and Sample Preparation}

2.3.1. Animal Experiment. Twelve male Sprague-Dawley rats $(200 \pm 20 \mathrm{~g})$ were purchased from the Laboratory Animal Center of Fudan University (Shanghai, China). All experiments on rats were implemented in accordance with the guidelines set by Shanghai Jiao Tong University Animal Ethics Committee (Shanghai, China). The animals were randomly divided into alantolactone group, isoalantolactone group, and blank group (each with four rats), housed under controlled conditions (temperature: $20-25^{\circ} \mathrm{C}$, humidity: $40-70 \%$ ) with a natural light-dark cycle for 1 week before the experiment. All rats were fasted overnight with water allowed ad libitum, and then a single dose of alantolactone or isoalantolactone was given orally to rats at $90 \mathrm{mg} / \mathrm{kg}$ (blank group received oral administration of water). Plasma samples were collected from the orbital vein at $60 \mathrm{~min}$ after administration [13]. Urine and feces samples from the rats were collected during $24 \mathrm{~h}$ after administration. A cannular port system was surgically implanted on the rats, which allowed continuous collection of bile. Bile samples from rats were obtained during $12 \mathrm{~h}$ after dosing. The samples were stored at $-20^{\circ} \mathrm{C}$ until further analysis.

2.3.2. Sample Preparation. Plasma sample $(100 \mu \mathrm{L})$ was added to $400 \mu \mathrm{L}$ acetonitrile in a $1.5 \mathrm{~mL}$ centrifuge tube. The tube was vortex-mixed for $1 \mathrm{~min}$ and centrifuged at 14,000 rpm for $10 \mathrm{~min}$. The supernatant $(2 \mu \mathrm{L})$ was injected into the LC-MS analysis.

Feces sample was pulverized with a mortar and pestle. Five volumes of normal saline and acetonitrile $(1: 3, \mathrm{w} / \mathrm{v})$ were added to $0.05 \mathrm{~g}$ pulverized feces and vortexed for $1 \mathrm{~min}$. After centrifugation at $14,000 \mathrm{rpm}$ for $10 \mathrm{~min}, 10 \mu \mathrm{L}$ supernatant was diluted with $1 \mathrm{~mL}$ acetonitrile. An aliquot of $2 \mu \mathrm{L}$ of the sample was injected into the LC-MS system for further analysis.

Urine sample $(100 \mu \mathrm{L})$ was added to $400 \mu \mathrm{L}$ acetonitrile in a $1.5 \mathrm{~mL}$ centrifuge tube. The tube was vortexed for $1 \mathrm{~min}$. After centrifugation at $14,000 \mathrm{rpm}$ for $10 \mathrm{~min}$, the supernatant was evaporated to dryness in a $40^{\circ} \mathrm{C}$ water bath by a nitrogen blowing instrument. The residue was reconstituted with $100 \mu \mathrm{L}$ mobile phase. All the dissolved samples were centrifuged at $14,000 \mathrm{rpm}$ for another $5 \mathrm{~min}$, and an aliquot of $2 \mu \mathrm{L}$ supernatant was injected into the LC-MS system for further analysis.

Bile sample $(15 \mu \mathrm{L})$ was added to $485 \mu \mathrm{L}$ acetonitrile in a $1.5 \mathrm{~mL}$ centrifuge tube, vortexed for $1 \mathrm{~min}$, and centrifuged at $14,000 \mathrm{rpm}$ for $10 \mathrm{~min}$. An aliquot of $2 \mu \mathrm{L}$ supernatant was injected into the LC-MS system for further analysis.

\subsection{Incubation In Vitro and Sample Preparation}

2.4.1. Incubation with Artificial Gastric Juice and Intestinal Juice. Two milligrams each of alantolactone or isoalantolactone was added to $10 \mathrm{~mL}$ of artificial gastric juice (containing $10 \mathrm{mg} / \mathrm{mL}$ pepsin and $0.08 \mathrm{M} \mathrm{HCl}, \mathrm{pH} 1.4$ ) and artificial intestinal juice (containing $10 \mathrm{mg} / \mathrm{mL}$ trypsin and $0.05 \mathrm{M}$ $\mathrm{KH}_{2} \mathrm{PO}_{4}, \mathrm{pH} 6.8$ ), respectively. The mixture was incubated at $37^{\circ} \mathrm{C}$ and one milliliter of incubated solution was transferred to a $10 \mathrm{~mL}$ volumetric centrifuge tube at $0,1,2,4$, and $6 \mathrm{~h}$ and adjusted to a $\mathrm{pH}$ of 7.0 with $0.1 \mathrm{M}$ sodium hydroxide. A fourfold volume of methanol was added to the incubated solution immediately and mixed. The mixture was centrifuged at $4800 \mathrm{rpm}$ for $20 \mathrm{~min}$, and the supernatant was evaporated under reduced pressure $\left(40^{\circ} \mathrm{C}\right)$ to produce a residue. The residue was dissolved in $1 \mathrm{~mL}$ of methanol, filtered through a $0.22 \mu \mathrm{m}$ membrane, and then analyzed by LC-MS.

2.4.2. Incubation with Rat Intestinal Bacteria. Fresh fecal samples from rats $(2 \mathrm{~g})$ were homogenized with 25 volumes of GAM broth. The sediments were removed by filtration through three pieces of gauze. The suspension was incubated at $37^{\circ} \mathrm{C}$ with $4 \mathrm{mg}$ alantolactone or isoalantolactone in an anaerobic incubator in which the air had been replaced with a gas mixture $\left(\mathrm{H}_{2} 5 \%, \mathrm{CO}_{2} 10 \%\right.$, and $\left.\mathrm{N}_{2} 85 \%\right)$ for $48 \mathrm{~h}$. The cultured mixture was taken out and extracted with watersaturated $n$-butanol at $0,6,12,24$, and $48 \mathrm{~h}$, respectively. The extract was then evaporated and the residue dissolved in $0.5 \mathrm{~mL}$ methanol. The redissolved sample was analyzed with LC-MS.

2.4.3. Incubation with Rat Microsomes. Samples for incubation were prepared with $50 \mu \mathrm{M}$ alantolactone or isoalantolactone, $5 \mathrm{mM} \mathrm{MgCl} 2,1 \mathrm{mM} \mathrm{NADPH}$, and $1 \mathrm{mg} / \mathrm{mL}$ microsomal protein in potassium phosphate buffer $(0.1 \mathrm{M}, \mathrm{pH}$ 7.4). After preincubation at $37^{\circ} \mathrm{C}$ for $3 \mathrm{~min}$, the incubations were initiated by the addition of NADPH and further incubated at $37^{\circ} \mathrm{C}$ for $60 \mathrm{~min}$ in a shaking water bath. The reactions were terminated with an equal volume of ice-methanol. The mixtures were then centrifuged at $12,000 \mathrm{rpm}$ for $10 \mathrm{~min}$ at $4^{\circ} \mathrm{C}$. The supernatant was analyzed for metabolite profiling by UPLC-MS.

2.5. UPLC-MS Conditions. Chromatographic separation was performed on an ACQUITY UPLC BEH $\mathrm{C}_{18}$ column $(100 \mathrm{~mm} \times 2.1 \mathrm{~mm}$ i.d, $1.7 \mu \mathrm{m}$, Waters Corp., Milford, MA, USA) using an ACQUITY ${ }^{\mathrm{TM}}$ UPLC system equipped with a 


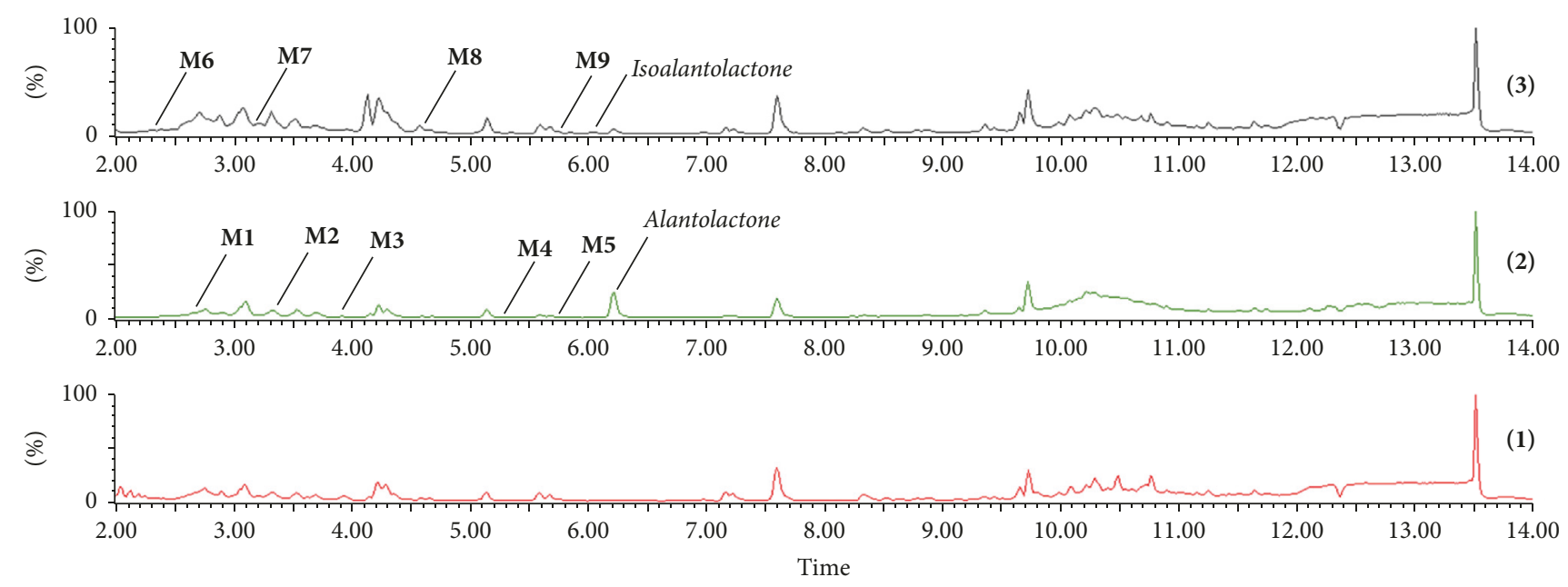

FIGURE 1: Representative TIC chromatograms of bile collected during $24 \mathrm{~h}$ after oral administration of alantolactone and isoalantolactone. (1) Blank group; (2) alantolactone group; and (3) isoalantolactone group.

binary pump, a DAD detector, a vacuum degasser, and an autosampler (Waters Corp., Milford, MA, USA). The detection wavelength was set at $210 \mathrm{~nm}$ and the column oven was maintained at $40^{\circ} \mathrm{C}$. The mobile phase contained acetonitrile (A) and $0.1 \%$ formic acid (B) solution, at a flow rate of $0.2 \mathrm{~mL} / \mathrm{min}$. For analysis of metabolites, the gradient system started with $5 \% \mathrm{~A}$, programmed linearly to $80 \% \mathrm{~A}$ in $20 \mathrm{~min}$, held for $2 \mathrm{~min}$, followed by a decrease to $20 \% \mathrm{~A}$ in $1 \mathrm{~min}$ to reequilibrate the column. The samples were kept at $10^{\circ} \mathrm{C}$ in the autosampler.

Mass spectrometry was carried out using a Waters Synapt mass spectrometer (Waters Corp., Milford, MA, USA). Ionization was operated using an ESI source in the positive ion mode. The ionization source parameters were as follows: capillary voltage $1 \mathrm{kV}$, cone voltage $30 \mathrm{~V}$, source temperature $110^{\circ} \mathrm{C}$, desolvation temperature $350^{\circ} \mathrm{C}$, desolvation gas flow $600 \mathrm{~L} / \mathrm{h}$, and cone gas flow $30 \mathrm{~L} / \mathrm{h}$. Nitrogen was used as the desolvation and cone gas, and argon was used as the collision gas. Data acquisition was performed in full-scan. All data acquired were processed using Masslynx v.4.1 software (Waters Corp., Milford, MA, USA).

\section{Results and Discussion}

3.1. Preparation of Alantolactone, Isoalantolactone, and Other Relevant Lactones. The repeated silica gel CC afforded alantolactone (1) and isoalantolactone (2). In addition, six relevant lactones 3,4-dehydroalantolactone (3), $5 \alpha, 6 \alpha$-epoxyalantolactone (4), 6-hydroxyl alantolactone (5), 4-hydroxymethyl isolalantolactone (6), 11-carboxyl alantolactone (7), and 11-carboxyl alantolactone (8) were obtained. Their structures were confirmed by MS and NMR determination [14-19].

3.2. The Metabolites Analysis of Alantolactone and Isoalantolactone In Vivo. The metabolites in plasma, bile, feces, and urine of rats orally administrated with alantolactone and isoalantolactone were investigated by UPLC-Q-TOF-MS analysis. Although no metabolites were detected in plasma, urine, and feces samples, 9 metabolites were found in bile samples (Figure 1 and Supplementary file available here). Their chemical structures were further deduced from their $t_{R}$ values, exact masses, formulas, and characteristic fragmentation ions (Table 1).

Metabolites M1 and M6 both with a formula of $\mathrm{C}_{15} \mathrm{H}_{20} \mathrm{O}_{4}$ were detected as protonated molecular ions $[\mathrm{M}+\mathrm{H}]^{+}$at $\mathrm{m} / z$ 265 which were $32 \mathrm{Da}$ higher than those of alantolactone and isoalantolactone, indicating that they could be peroxidation products of alantolactone and isoalantolactone. Product ions at $m / z 247$ were also observed, which suggested the successive loss of $\mathrm{H}_{2} \mathrm{O}$ through the cleavage of the lactone ring. $\mathrm{M} 1$ and M6 were further confirmed to be 11-carboxyl alantolactone and 11-carboxyl isoalantolactone, by their identical retention times and the same molecular weights to compounds 7 and 8 , respectively.

Metabolites M2 and M7 both with a formula of $\mathrm{C}_{18} \mathrm{H}_{27} \mathrm{NO}_{4} \mathrm{~S}$ exhibited $[\mathrm{M}+\mathrm{H}]^{+}$ions at $\mathrm{m} / z$ 354, which were $119 \mathrm{Da}$ higher than those of alantolactone and isoalantolactone, indicating that M2 and M7 were their cysteine conjugates. The presence of a minor fragment ion at $\mathrm{m} / z 120$ further indicated the existence of cysteine. Based on these results and the findings from the previous report [20], M2 and M7 were considered as the two-phase reaction metabolites of liver and speculated to be 13-cysteine alantolactone and 13cysteine isoalantolactone, respectively.

Metabolite M3 with a retention time of $3.898 \mathrm{~min}$ and a formula of $\mathrm{C}_{15} \mathrm{H}_{20} \mathrm{O}_{3}$ was observed as protonated molecular ion $[\mathrm{M}+\mathrm{H}]^{+}$at $m / z 249$ which was 16 Da higher than the protonated ion of alantolactone, indicating that M3 was an epoxide of alantolactone. The MS spectrum of M3 also showed a product ion at $m / z 231$, which was generated from $m / z$ 249 by loss of $\mathrm{H}_{2} \mathrm{O}$. M3 was further identified as $5 \alpha, 6 \alpha-$ epoxyalantolactone by its identical retention time and molecular weight to compound 4.

Metabolite M4 was observed as protonated molecular ion $[\mathrm{M}+\mathrm{H}]^{+}$at $m / z 231$, which was $2 \mathrm{Da}$ lower than the 


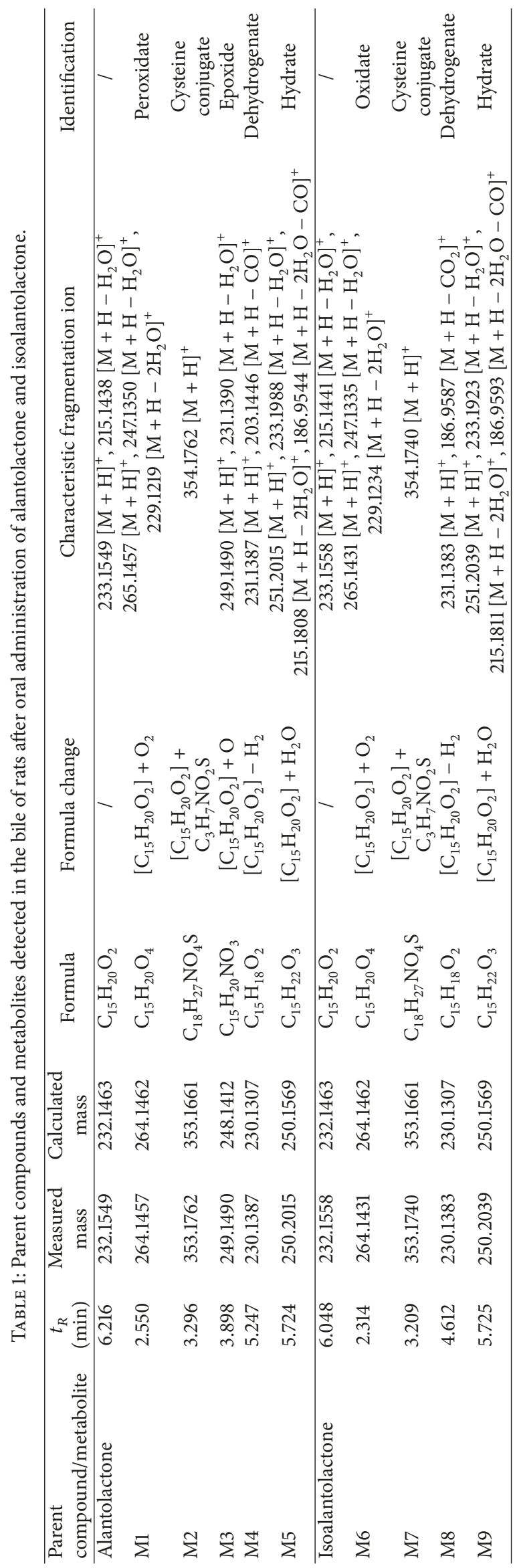




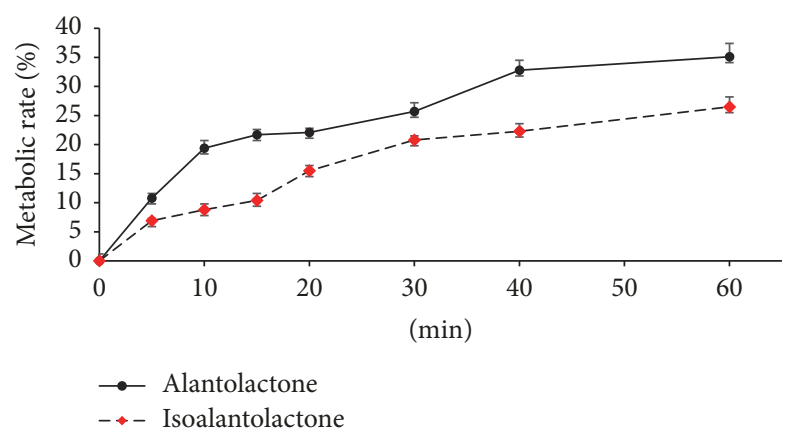

FIGURE 2: Metabolism profiles of alantolactone and isoalantolactone incubated with rat liver microsomes. Data are presented as mean $\pm \mathrm{SD}$, $n=3$. Metabolic ratio $=\left(P_{0}-P_{t}\right) / P_{0} \times 100 \%, P_{0}$ : peak area of 0 min; $P_{t}$, peak area of $t$ min.
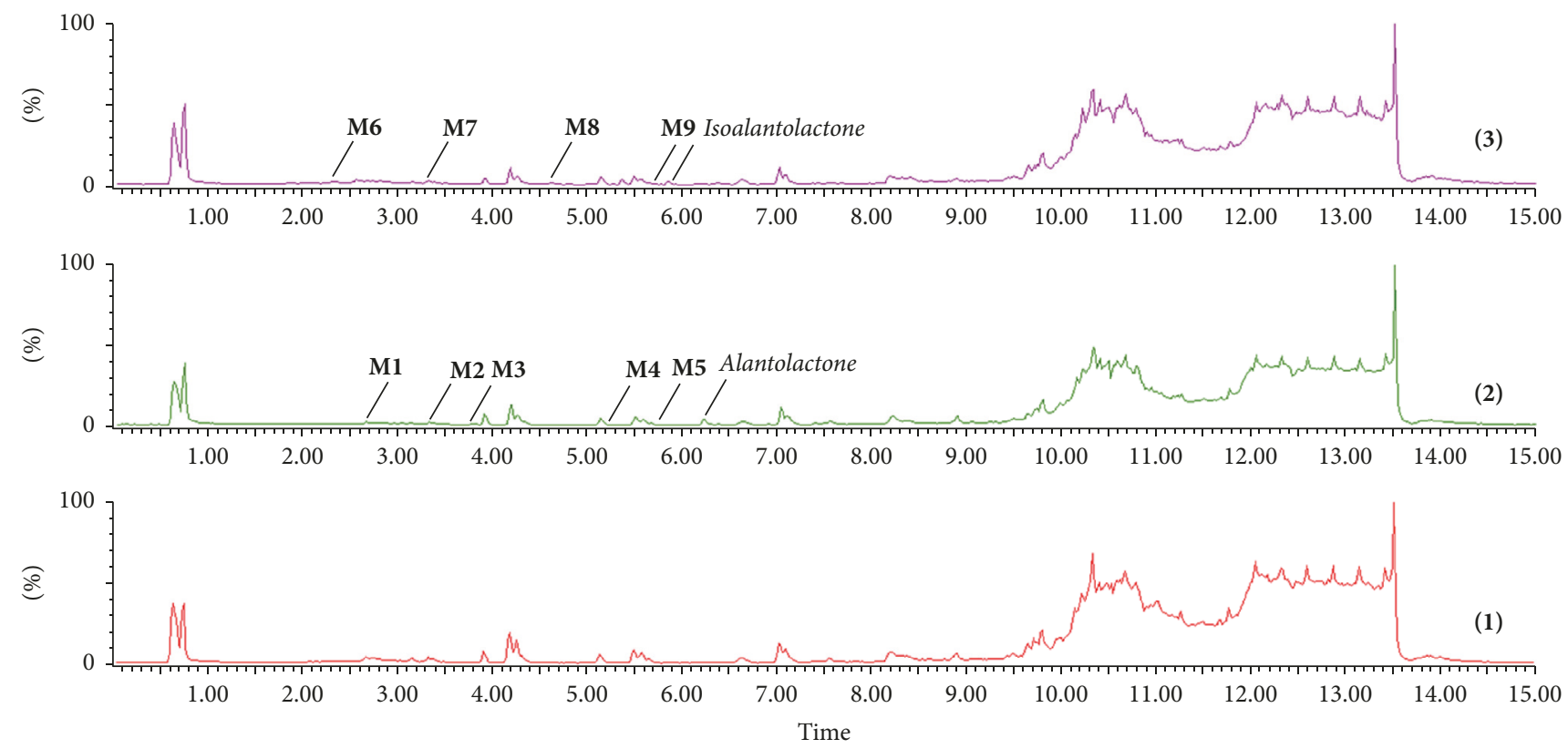

FIGURE 3: The representative TIC chromatograms of alantolactone and isoalantolactone incubated with rat liver microsomes for $1 \mathrm{~h}$. (1) Blank; (2) alantolactone; and (3) isoalantolactone; M1-M9, metabolites.

protonated ion of alantolactone, indicating that M4 was a dehydrogenate. The MS spectrum also showed a product ion at $m / z 203$, which was generated from $m / z 231$ by loss of CO. M4 was confirmed to be 3,4-dehydroalantolactone by the identical retention time and the same molecular weight to compound 3.

Metabolites M5 and M9 were detected as protonated molecular ions $[\mathrm{M}+\mathrm{H}]^{+}$at $m / z 251$, which were 18 Da higher than the protonated ions of alantolactone and isoalantolactone, indicating that M5 and M9 could be their hydrates. MS spectra also showed fragment ions at $m / z 233$, corresponding to $\left[\mathrm{M}+\mathrm{H}-\mathrm{H}_{2} \mathrm{O}\right]^{+}$, suggesting the presence of a hydroxyl group. M5 and M9 were confirmed to be 6-hydroxyl alantolactone and 15-hydroxyl isoalantolactone by the identical retention times and molecular weights to compounds 5 and 6, respectively.

Metabolite M8 was observed at $m / z 231[\mathrm{M}+\mathrm{H}]^{+}$and also considered as a dehydrogenate. The MS spectrum showed a product ion at $m / z 203$, which was generated from $m / z 231$ by loss of CO. Based on these results and the findings from our previous research [21], M8 was speculated to be 5, 6dehydroisoalantolactone.

3.3. The Metabolic Organ of Alantolactone and Isoalantolactone. The results mentioned above suggested that liver was the main metabolic organ of alantolactone and isoalantolactone. To confirm this result, alantolactone and isoalantolactone each was incubated in vitro with gastric juice, intestinal juice, fecal bacteria, and liver microsomes. The lactones were found to be stable in gastric juice, intestinal juice, and fecal bacteria, respectively. However, the incubation of alantolactone and isoalantolactone with the liver microsomes from the experimental animal led to the rapid metabolism (Figure 2) and generated 9 metabolites identical to those found in vivo (Figure 3). This confirmed that the main 


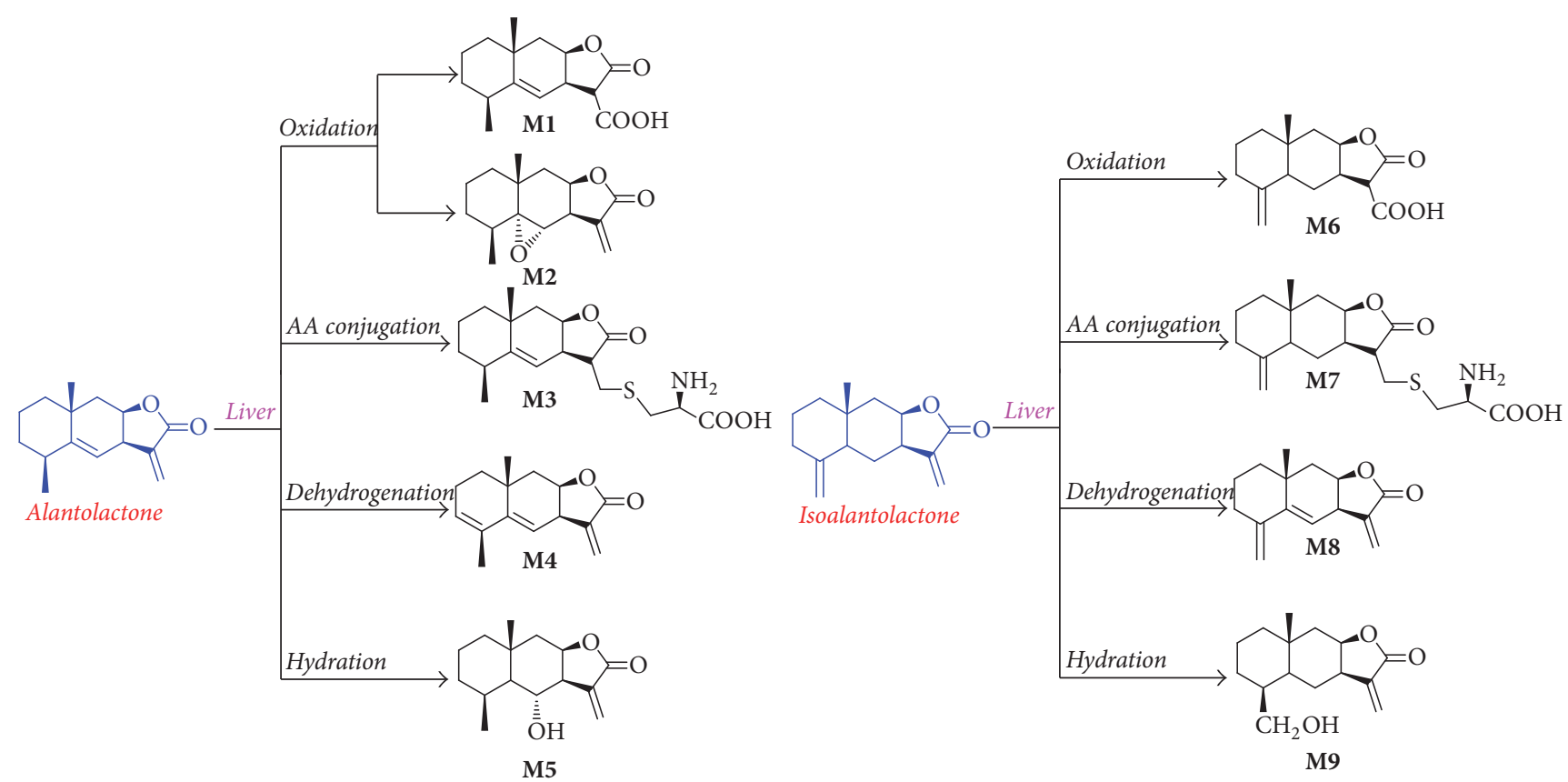

FIgUre 4: Possible metabolic pathways of alantolactone and isoalantolactone in rat.

metabolic organ for the two lactones was the liver. Possible metabolic pathways for the lactones are shown in Figure 4.

\section{Conclusion}

In the present study, metabolism of alantolactone and isoalantolactone in vivo (urine, feces, bile, and plasma) and in vitro (gastric juice, intestinal juice, fecal bacteria, and liver microsomes) were investigated. Nine metabolites were found in bile and liver microsomes, indicating that a first pass effect of the liver on the two lactones existed.

\section{Conflicts of Interest}

The authors declare that there are no conflicts of interest regarding the publication of this paper.

\section{Acknowledgments}

This study was financially supported by the National Science \& Technology Key Projects funded by the Government of China (2012ZX09103201-038) and National Natural Science Fund of China (no. 81374067). The authors are really grateful to Miss Jieli Wu of the Instrumental Analysis Center of Shanghai Jiao Tong University for the measurements of NMR.

\section{Supplementary Materials}

Figure 1: HR ESI (+) MS spectrum of alantolactone detected in bile. Figure 2: HR ESI (+) MS spectrum of alantolactone metabolite M1 detected in bile. Figure 3: HR ESI (+) MS spectrum of alantolactone metabolite M2 detected in bile.
Figure 4: HR ESI (+) MS spectrum of alantolactone metabolite M3 detected in bile. Figure 5: HR ESI (+) MS spectrum of alantolactone metabolite M4 detected in bile. Figure 6: HR ESI (+) MS spectrum of alantolactone metabolite M5 detected in bile. Figure 7: HR ESI (+) MS spectrum of isoalantolactone detected in bile. Figure 8: HR ESI (+) MS spectrum of isoalantolactone metabolite M6 detected in bile. Figure 9: HR ESI (+) MS spectrum of isoalantolactone metabolite M7 detected in bile. Figure 10: HR ESI (+) MS spectrum of isoalantolactone metabolite M8 detected in bile. Figure 11: HR ESI (+) MS spectrum of isoalantolactone metabolite M9 detected in bile. (Supplementary Materials)

\section{References}

[1] F. Bohlmann, P. K. Mahanta, J. Jakupovic, R. C. Rastogi, and A. A. Natu, "New sesquiterpene lactones from Inula species," Phytochemistry, vol. 17, no. 7, pp. 1165-1172, 1978.

[2] Editorial Board of Chinese Pharmacopoeia, Pharmacopoeia of the People's Republic of China, China Medical Science Press, Beijing, China, 2015.

[3] Z. Stojanović-Radić, L. Čomić, N. Radulović et al., "Antistaphylococcal activity of Inula helenium L. root essential oil: eudesmane sesquiterpene lactones induce cell membrane damage," European Journal of Clinical Microbiology \& Infectious Diseases, vol. 31, no. 6, pp. 1015-1025, 2012.

[4] J. Qiu, M. Luo, J. Wang et al., "Isoalantolactone protects against Staphylococcus aureus pneumonia," FEMS Microbiology Letters, vol. 324, no. 2, pp. 147-155, 2011.

[5] M. Khan, C. Ding, A. Rasul et al., "Isoalantolactone induces reactive oxygen species mediated apoptosis in pancreatic carcinoma PANC-1 cells," International Journal of Biological Sciences, vol. 8, no. 4, pp. 533-547, 2012.

[6] W. Wei, H. Huang, and S. Zhao, "Alantolactone induces apoptosis in chronic myelogenous leukemia sensitive or resistant 
to imatinib through $\mathrm{NF}-\kappa \mathrm{B}$ inhibition and $\mathrm{Bcr} / \mathrm{Abl}$ protein deletion," Apoptosis, vol. 18, no. 9, pp. 1060-1070, 2013.

[7] A. Rasul, M. Khan, B. Yu et al., "Isoalantolactone, a sesquiterpene lactone, induces apoptosis in SGC-7901 cells via mitochondrial and phosphatidylinositol 3-kinase/Akt signaling pathways," Archives of Pharmacal Research, vol. 36, no. 10, pp. 1262-1269, 2013.

[8] J. Chun, R.-J. Li, M.-S. Cheng, and Y. S. Kim, "Alantolactone selectively suppresses STAT3 activation and exhibits potent anticancer activity in MDA-MB-231 cells," Cancer Letters, vol. 357, no. 1, pp. 393-403, 2015.

[9] A. Rasul, J. Di, F. M. Millimouno et al., "Reactive oxygen species mediate isoalantolactone-induced apoptosis in human prostate cancer cells," Molecules, vol. 18, no. 8, pp. 9382-9396, 2013.

[10] A. Rasul, M. Khan, M. Ali, J. Li, and X. Li, "Targeting apoptosis pathways in cancer with alantolactone and isoalantolactone," The Scientific World Journal, vol. 2013, Article ID 248532, 2013.

[11] Y. Peng, S. Wang, M. Wang et al., "Dual effects on constipation and diarrhea: Protective potential of Radix Inulae lactones on irritable bowel syndrome," RSC Advances, vol. 6, no. 97, pp. 94486-94495, 2016.

[12] R. Xu, G. Zhou, Y. Peng, M. Wang, and X. Li, "Pharmacokinetics, tissue distribution and excretion of isoalantolactone and alantolactone in rats after oral administration of Radix Inulae Extract," Molecules, vol. 20, no. 5, pp. 7719-7736, 2015.

[13] R. Xu, M. Wang, Y. Peng, and X. Li, "Pharmacokinetic comparison of isoalantolactone and alantolactone in rats after administration separately by optimization of an UPLC-MS2 method," Journal of Chemistry, vol. 2014, Article ID 354618, 2014.

[14] H. J. Du, H. M. Shi, M. Y. Wang, C. S. Peng, and X. B. Li, "Structural modification of isoalantolactone and alantolactone isolated from," in Inula helenium, vol. 25, pp. 156-160, Natural Product Research and Development, 2013.

[15] S. G. Klochkov, S. V. Afanaséva, and A. N. Pushin, "Acidic isomerization of alantolactone derivatives," Chemistry of Natural Compounds, vol. 42, no. 4, pp. 400-406, 2006.

[16] R. S. Dhillon, K. Nayyar, S. Singh, and Z. S. Dhaliwal, "Chemical transformations of alpha-methylene-gamma-lactones of Parthenium hysterophorus Linn. and Inula racemosa Hook. and ovicidal activity of products against maize borer, Chilo partellus," Indian Journal of Chemistry, vol. 14, no. 8, pp. 35243530, 1994.

[17] Y. Huo, H. Shi, M. Wang, and X. Li, "Complete assignments of1H and13C NMR spectral data for three sesquiterpenoids from Inula helenium," Magnetic Resonance in Chemistry, vol. 46, no. 12, pp. 1208-1211, 2008.

[18] A. G. Schultz and J. D. Godfrey, "An annelation approach to the synthesis of eudesmane and elemane sesquiterpene lactones. Total synthesis of dl-dihydrocallitrisin, dl-7,8-epialantolactone, dl-7,8-epiisoalantolactone, and dl-atractylon," Journal of the American Chemical Society, vol. 102, no. 7, pp. 2414-2428, 1980.

[19] Q. He, X. C. Liu, R. Q. Sun, Z. W. Deng, S. S. Du, and Z. L. Liu, "Mosquito larvicidal constituents from the ethanol extract of inula racemosa Hook. f. roots against Aedes albopictus," Journal of Chemistry, vol. 2014, Article ID 738796, 2014.

[20] G. Dupuis, J. C. Mitchell, and G. H. N. Towers, "Reaction of alantolactone, an allergenic sesquiterpene lactone, with some amino acids. Resultant loss of immunologic reactivity," Canadian Journal of Biochemistry, vol. 52, no. 7, pp. 575-581, 1974.
[21] Y. Huo, H. M. Shi, M. Y. Wang, and X. B. Li, "Chemical constituents and pharmacological properties of Radix Inulae," Die Pharmazie, vol. 63, no. 10, pp. 699-703, 2008. 

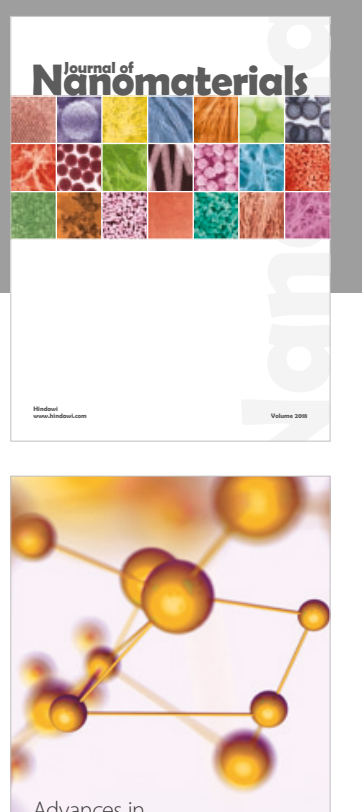

Physical Chemistry
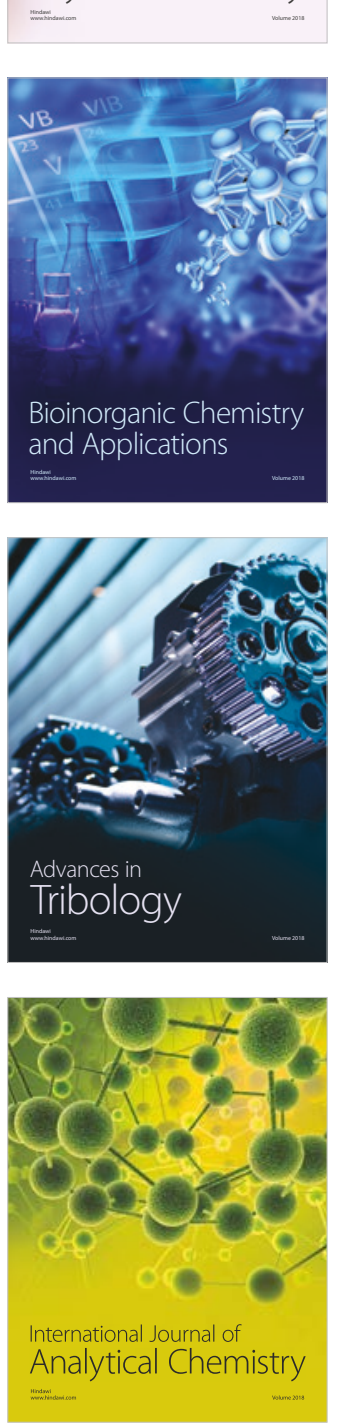

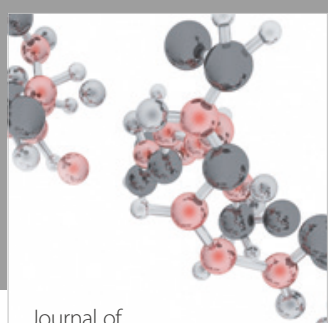

Analytical Methods

in Chemistry

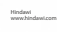

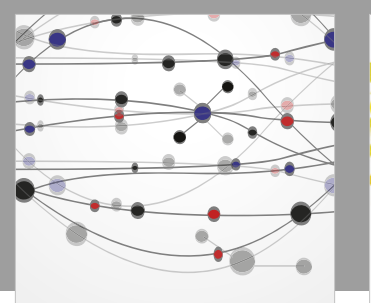

The Scientific World Journal

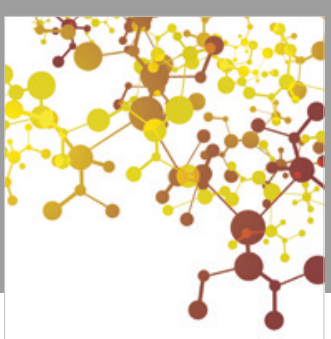

Journal of

Applied Chemistry
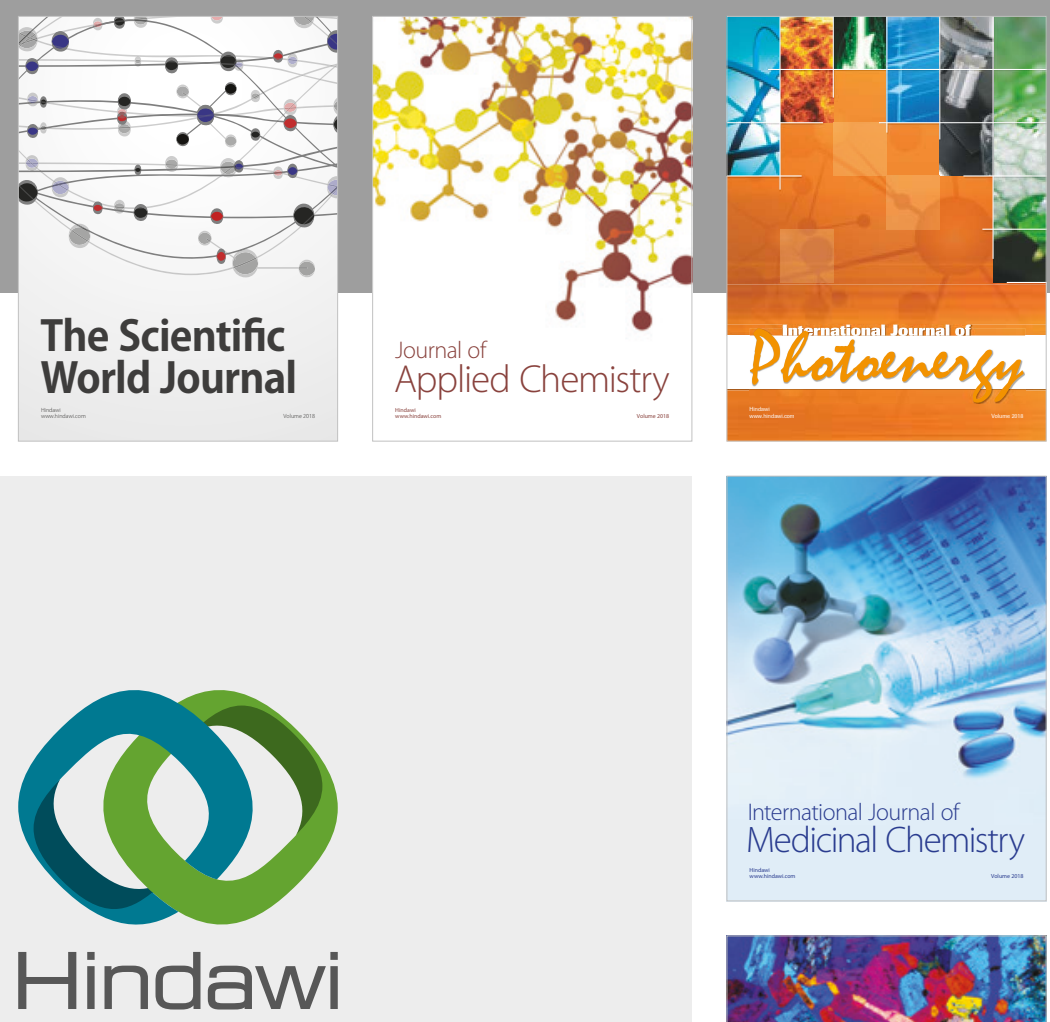

Submit your manuscripts at

www.hindawi.com
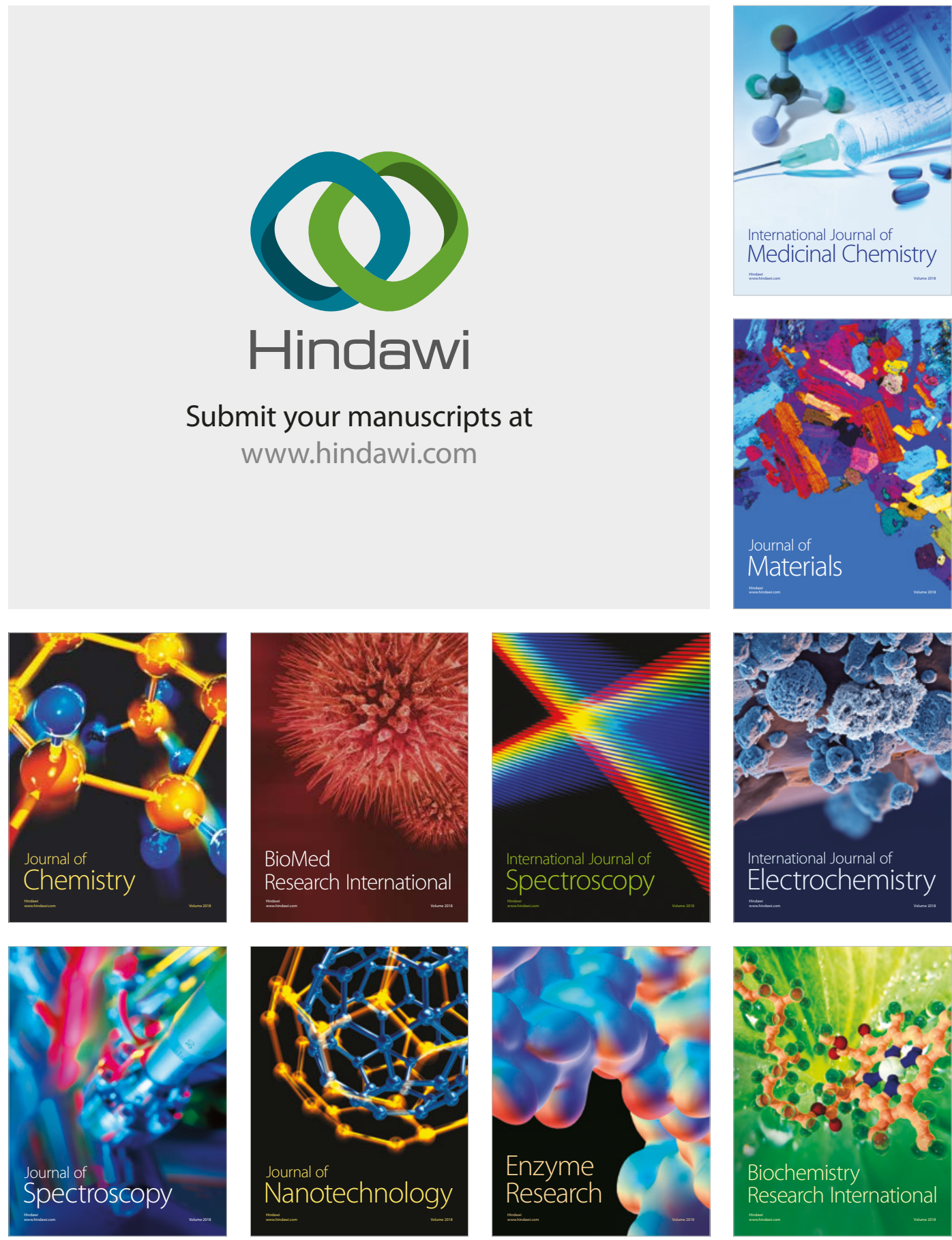
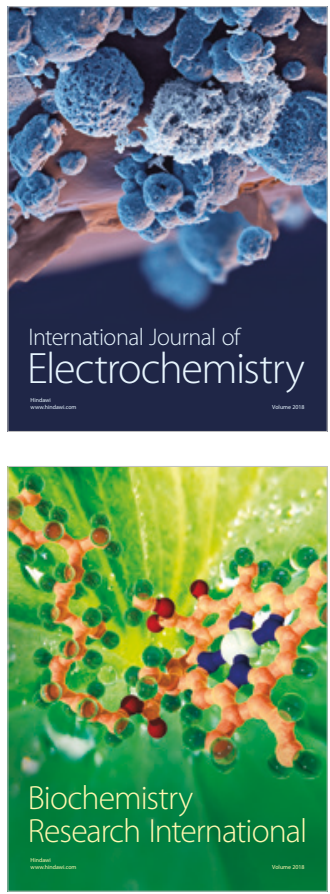